determine if hearing loss treatment versus a successful aging control intervention can reduce the risk of cognitive decline in older adults. This trial will be nested within ARIC, and a pilot study was recently completed that established trial feasibility and intervention efficacy. In this 6-month pilot study of 40 individuals aged 70-84 years, the hearing intervention demonstrated a clear efficacy signal on communication and social functioning (domains hypothesized to mediate downstream effects of HL on cognitive decline). Estimated changes in standardized (z-score) outcomes were qualitatively different by intervention assignment for all measures, including perceived handicap due to hearing loss (HHIE, $\mathrm{p}<0.0001$ ), loneliness, number of contacts $(\mathrm{p}=0.007)$ and diversity of social network, and social, mental, and physical function.

\section{APPLICATION OF LATENT VARIABLE METHODS TO THE STUDY OF COGNITIVE DECLINE WHEN TESTS CHANGE OVER TIME}

A. Gross ${ }^{1,2}$, S. Burgard 3 , S. Davis ${ }^{3}$, J.A. Deal ${ }^{1,2}$, T.H. Mosley ${ }^{4}$, J. Coresh ${ }^{1}$, A. Sharrett ${ }^{1}$, 1. Department of Epidemiology, Johns Hopkins Bloomberg School of Public Health, Baltimore, Maryland, 2. Johns Hopkins University Center on Aging and Health, Baltimore, Maryland, 3. Department of Biostatistics, UNC Gillings School of Global Public Health, Chapel Hill, North Carolina, 4. Department of Medicine, University of Mississippi Medical Center, Jackson, Mississippi

We demonstrated the use of factor analysis to link differing cognitive batteries over ARIC-NCS visits to common metrics representing general and domain-specific cognition. We used 23 years of data from the Atherosclerosis Risk in Communities Study (ARIC-NCS) (N=14,252). Using generalized estimating equations, we compared associations of diabetes with cognitive change using general and domainspecific factor scores vs. z- scores. Factor scores provided stronger associations with diabetes at the expense of greater variability around estimates (e.g., for executive functioning, -0.065 SD units/year, $\mathrm{SE}=0.015$, vs -0.057 SD units/year, $\mathrm{SE}=0.013$ ), suggesting factor scores more explicitly address error in measured traits. We calibrated general and domainspecific cognitive performance across study visits in which different but overlapping cognitive tests were administered at each visit. Factor analysis facilitates use of all available data when measures change over time. We further demonstrate how to estimate factor scores during real-time data collection to enable selection into further screening.

\section{SESSION 345 (PAPER)}

\section{MULTI-MORBIDITY AND ENVIRONMENTAL FACTORS AFFECTING GAIT AND FUNCTION}

\section{MULTI-MORBIDITY PATTERNS AND DISABLEMENT SEVERITY AMONG MOBILITY LIMITED OLDER ADULTS}

M.E. Jacob ${ }^{1,2,3}$, P. Ni ${ }^{1}$, E. Leritz ${ }^{2,3}$, J. Driver ${ }^{2,3}$, S.G. Leveille ${ }^{4}$, A.M. Jette ${ }^{1}$, J.F. Bean ${ }^{2,3}, 1$. Health and Disability Research Institute, Boston University, Brookline, Massachusetts, 2. New England GRECC, VA Boston Healthcare System, Boston, Massachusetts, 3. Harvard Medical School, Boston,
Massachusetts, 4. University of Massachusetts Boston, Boston, Massachusetts

Multi-morbidity contributes to functional decline among older adults. However, it is not evident whether specific patterns of multi-morbidity are associated with worse disablement outcomes. We analyzed baseline data from the Boston RISE study, a cohort of older primary care patients with mobility limitations, to examine the association between multi-morbidity patterns and neuromuscular impairments and function. Participants $(\mathrm{n}=425)$ self-reported 13 major chronic conditions and underwent assessment of impairments (leg strength, leg velocity, trunk extensor endurance, leg range of motion, sensation) and function $(400 \mathrm{~m}$ walk test, Short Physical Performance Battery (SPPB), Late Life Function and Disability Instrument (LLFDI)). We conducted Latent Class Analysis among the chronic conditions and examined impairments and functional status among different latent classes. LCA identified a high multi-morbidity group with high prevalence of cardiovascular, metabolic and musculoskeletal diseases (Class 1), a low multi-morbidity group (Class 2) and a musculoskeletal group with high prevalence of arthritis, back pain and osteoporosis, but few other conditions (Class 3). After adjusting for age and gender, Class 1 had significantly lower strength, range of motion, SPPB score, gait speed and LLFDI scores compared to Class 2. However, Class 3 demonstrated less range of motion impairment and similar SPPB, gait speed and LLFDI scores compared to Class 2 but similar pain levels to Class 1. Among mobility limited older adults, a sub group with predominantly musculoskeletal conditions did not have worse neuromuscular impairments or function than a healthier sub-group; the sub-group with co-occurring musculoskeletal conditions and other comorbidities had the worst impairments and function.

\section{IS NEURAL CONTROL OF WALKING IMPORTANT BEYOND GAIT SPEED?}

J.M. Van Swearingen, C. Smith, L. Coffman, S. Perera, J. Brach, University of Pittsburgh, Pittsburgh, Pennsylvania

Gait speed is a robust index of walking and functioning, but may not represent neural control of walking. Integrating spatial and temporal aspects of gait, the walk ratio (WR, step length/step rate) is a measure of neural control and is associated with energy expenditure. We explored the WR in relation to mobility-related endurance (6-minute walk. 6MWD) and walking confidence (modified Gait Efficacy Scale) in older adult participants in a community-based trial $(n=391$; mean age, $80.5 \pm 7.7$ years). Gait speed was $0.92 \pm 0.21 \mathrm{~m} / \mathrm{s}$ and WR $0.0048 \pm 0.0008 \mathrm{~m} / \mathrm{steps} / \mathrm{min}$. Adjusting for gait speed, a $0.001 \mathrm{~m} / \mathrm{steps} / \mathrm{min}$ difference in WR was associated with 15 meters in 6MWD and 2.5 points in confidence (both $\mathrm{p}<0.005)$. A clear gradient of mobility-related performance across WR quartiles was observed within quartiles of gait speed. Those who walked within the narrow gait speed range of $0.77-0.90 \mathrm{~m} / \mathrm{s}, 6 \mathrm{MWD}$ ranged $237-281$ meters and confidence ranged 71.1-77.1 points across WR quartiles. Among those with gait speed $>1.04 \mathrm{~m} / \mathrm{s}, 6 \mathrm{MWD}$ ranged $317-369$ meters and confidence $75.8-84.1$ points across WR quartiles. Good and poor neural control of walking are possible at both slower and faster gait speed, and is associated with endurance and confidence independent of gait speed. Neural control of walking is an important aspect of gait and should 